\title{
Cecal volvulus associated with mobile cecum in Cornelia de Lange syndrome: Report of a case*
}

\author{
Masataka Takahashi ${ }^{1}$, Toshihiko Watanabe ${ }^{1}$, Hideaki Tanaka ${ }^{1}$, Waka Yamada ${ }^{1}$, Koji Yamada $^{1}$, \\ Yasushi Fuchimoto $^{1}$, Shunsuke Nosaka ${ }^{2}$, Yutaka Kanamori ${ }^{1}$ \\ ${ }^{1}$ Division of Surgery, Department of Surgical Subspecialties, National Center for Child Health and Development, Tokyo, Japan \\ ${ }^{2}$ Division of Radiology, Department of Radiology, National Center for Child Health and Development, Tokyo, Japan \\ Email: kanamori-y@ncchd.go.jp
}

Received 20 April 2013; revised 19 May 2013; accepted 27 May 2013

Copyright @ 2013 Masataka Takahashi et al. This is an open access article distributed under the Creative Commons Attribution License, which permits unrestricted use, distribution, and reproduction in any medium, provided the original work is properly cited.

\begin{abstract}
We report a case of cecal volvulus in an 11-year-old girl who had been diagnosed with Cornelia de Lange syndrome. She had undergone operative fundoplication several years earlier and was referred to our institute for treatment of intestinal obstruction. A severely dilated colon was detected on abdominal roentgenogram, and abdominal CT and colonic enema strongly suggested cecal volvulus. Emergency operation was performed and cecal volvulus with mobile cecum was ascertained; the volvulus was corrected and the mobile cecum was fixed to the peritoneum on the right side. Neurologically handicapped patients sometimes suffer from cecal volvulus, and patients with Cornelia de Lange syndrome are at especially high risk. Moreover, operative fundoplication promotes intestinal dilatation and it may be another risk factor for cecal volvulus.
\end{abstract}

Keywords: Cecal Volvulus; Cornelia de Lange Syndrome; Whirl Sign

\section{INTRODUCTION}

Cecal volvulus is a rare cause of intestinal obstruction and is often seen in neurologically handicapped patients [1]. Cecal volvulus is not easy to diagnose in many cases and severe necrosis of the affected portion of the intestine may result in the patient's death. Several studies reported the characteristic features of cecal volvulus on radiographic examinations such as barium enema and abdominal computed tomography (CT) [2-5], but in many cases preoperative diagnosis is difficult.

Cecal volvulus is also rare among pediatric patients and is often seen in neurologically handicapped patients

\footnotetext{
*The authors have no conflict of interest.
}

[6]. Patients with Cornelia de Lange syndrome are at especially high risk for cecal volvulus [7-11].

Here we report a case of cecal volvulus in a pediatric patient with Cornelia de Lange syndrome, who was diagnosed with cecal volvulus by CT findings and colonic enema before surgical treatment.

\section{CASE}

An 11-year-old female patient with Cornelia de Lange syndrome was referred to our institute because of pale face and loss of activity. She had undergone surgical fundoplication several years earlier for gastroesophageal reflux. Plain abdominal roentgenogram showed prominent dilatation of the stomach and intestinal loop in the upper abdomen (Figure 1(a)).

Drainage of stomach gas was performed via nasogastric tube and the symptoms subsided transiently. However, one day later, the drainage fluid from the nasogastric tube contained bile-like yellowish fluid. We performed diagnostic examinations. Abdominal CT demonstrated extremely dilated colon at the upper abdomen (Figure 1(b)), but the descending and sigmoid colon were decompressed. The typical whirl sign was seen in the lower left portion of the abdomen (Figures 1(c) and (d)). These findings strongly suggested that the patient suffered from colonic volvulus. The dilated colon was well contrasted upon administration of the intravenous contrast medium and bowel ischemia was not suggested. We then performed colonic enema to ascertain which portion of the colon was affected by volvulus. The rectum, sigmoid colon, descending colon and transverse colon were not dilated and the bird beak sign appeared just next to the transverse colon (Figure 1(e)), and we concluded that cecal volvulus was the cause of intestinal obstruction in this patient.

Emergency operation was performed and revealed cecal 

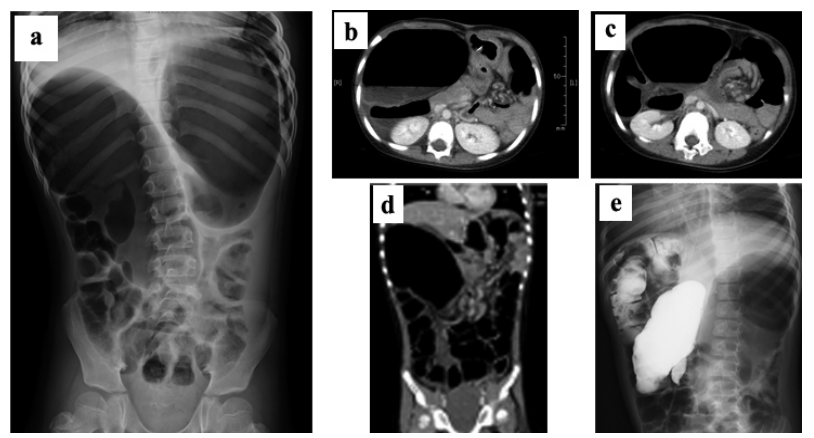

Figure 1. (a) Abdominal plain roentgenogram taken at admission. Prominently dilated stomach in the upper left side and abnormal intestinal dilatation in the upper right side of the abdomen were detected; (b) Abdominal contrast-enhanced CT. Dilated intestine that seemed to be a dilated colon was seen. Distal colon decompression was detected and these findings strongly suggested cecal volvulus; (c), (d) Abdominal contrastenhanced CT. Whirl sign was detected in the abdomen on the left side, which usually suggests sigmoid colon volvulus. However, this case was complicated with mobile cecum and the whirl sign had moved to the left side; (c), transverse image; (d), frontal image; (e) Colonic enema showed decompressed rectum, decompressed sigmoid, descending, and transverse colon, and bird beak sign in the abdomen on the right side. This film strongly suggested cecal volvulus and it was ascertained at the laparotomy.

volvulus. The cecum showed a $360^{\circ}$ clockwise rotation (axial volvulus), but bowel ischemia did not occur in the affected intestine (Figure 2(a)). The cecum and ascending colon were not fixed to the retroperitoneum and were so-called mobile cecum (Figure 2(b)). The rotation was corrected and the ascending and cecal colon was adhered to the peritoneum at the right side by absorbable sutures. The postoperative course was uneventful.

\section{DISCUSSION}

Cecal volvulus is a very rare cause of intestinal obstruction in all ages and is often seen in patients with a neurological disorder [1]. It is quite difficult to diagnose cecal volvulus in its early stage. In many cases, intestinal necrosis and perforation have already occurred at the time of diagnosis and in such cases the disease has become life-threatening.

It is very important to keep in mind that patients with Cornelia de Lange syndrome often have incomplete fixation of the cecum and sometimes suffer from cecal volvulus. Six cases of Cornelia de Lange syndrome in the English literature and the present case developed cecal volvulus [7-11]. We must also be aware that neurologycally disabled patients such as patients with trisomy 18 or cerebral palsy may suffer from cecal volvulus as well $[6,11]$. Moreover, we must remember that cecal volvulus occurred in some cases that had undergone operative fundoplication. Increased intestinal air due to massive

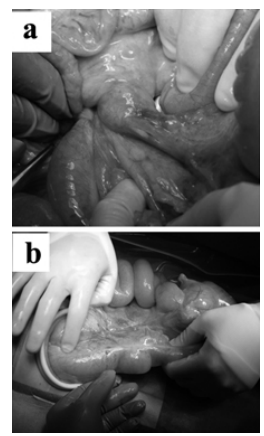

Figure 2. Operative findings. (a) The cecum was rotated $360^{\circ}$ clockwise and the blood supply was well maintained. (b) Mobile cecum was ascertained after correcting the rotation.

airophagy and anti-reflux operation might be a cause of volvulus in these cases. Four out of the seven cases with Cornelia de Lange syndrome experienced fundoplication before cecal volvulus including our case [11].

Another important feature in cecal volvulus is diagnostic images. Characteristic findings including those on plain abdominal roentgenogram and barium enema have been reported in several papers [2-4]. Among them, CT findings may be the most important. Distal colon decompression may be suggestive of the disease and a whirl sign is usually seen in the abdomen on the right side, whereas a whirl sign seen in the abdomen in the middle to left side is suggestive of sigmoid colon volvulus [4]. However, in our case distal colon decompression was ascertained but the whirl sign was seen in the abdomen on the left side. We must carefully look for the whirl sign of cecal volvulus in CT images in cases with mobile cecum because the whirl sign may be demonstrated in the abdomen on the left side.

We must suspect cecal volvulus when intestinal obstruction is seen in neurologically disabled patients who underwent fundoplication. In such cases, abdominal CT may be the most important diagnostic procedure.

\section{REFERENCES}

[1] Takeuchi, H., Ikeda, Y., Komori, Y., Tahara, K., Shiromizu, A., Hayashi, H., et al. (2008) Cecal volvulus in cerebral palsy: Report of a case. Surgery Today, 38, 170173. doi:10.1007/s00595-007-3581-x

[2] Consorti, E.T. and Liu, T.H. (2005) Diagnosis and treatment of caecal volvulus. Postgraduate Medical Journal, 81, 772-776. doi:10.1136/pgmj.2005.035311

[3] Rosenblat, J.M., Rozenblit, A.M., Wolf, E.L., DuBrow, R.A., Den, E.I. and Levsky, J.M. (2010) Findings of cecal volvulus at CT. Radiology, 256, 169-175. doi:10.1148/radiol.10092112 
[4] Macari, M., Spieler, B., Babb, J. and Pachter, H.L. (2011) Can the location of the CT whirl sign assist in differentiating sigmoid from caecal volvulus? Clinical Radiology, 66, 112-117. doi:10.1016/j.crad.2010.09.010

[5] Lee, N.K., Kim, S., Jeon, T.Y., Kim, H.S., Kim, D.H., Seo, H.I., et al. (2010) Complications of congenital and developmental abnormalities of the gastrointestinal tract in adolescents and adults: Evaluation with multimodality imaging. Radiographics, 30, 1489-1507. doi:10.1148/rg.306105504

[6] Phillips, G. and Parisi, M.T. (2010) Cecal volvulus: Classic imaging appearance of an uncommon pediatric entity. Pediatric Radiology, 40, S98. doi:10.1007/s00247-010-1588-8

[7] Hart, Z.H., Jaslow, R.I. and Gomez, M.R. (1965) The de Lange syndrome. American Journal of Diseases of Children, 109, 325-332.
[8] Husan, K., Fitzgerald, P. and Lau, G. (1994) Cecal volvulus in the Cornelia de Lange syndrome. Journal of Pediatric Surgery, 29, 1245-1247. doi:10.1016/0022-3468(94)90814-1

[9] Frischman, W.J., Couper, R.T.L. and Freeman, J.K. (1996) Cecal volvulus following gastroduodenoscopy in Cornelia de Lange syndrome. Journal of Pediatric Gastroenterology and Nutrition, 22, 205-207. doi:10.1097/00005176-199602000-00013

[10] Masumoto, K., Izaki, T. and Arima, T. (2001) Cornelia de Lange syndrome associated with cecal volvulus: Report of a case. Acta Paediatrica, 90, 701-703. doi:10.1080/080352501750258801

[11] Takeda, K., Hamada, Y., Sato, M., Fujii, Y., Teraguchi, M., Kaneko, K., et al. (2007) Cecal volvulus in children with mental disability. Pediatric Surgery International, 23, 1011-1014. doi:10.1007/s00383-007-1987-6 